\title{
Correlation between Rainfall and Cold Fronts with Gamma Ray Measurements near Ground Level in Southeastern Brazil
}

\author{
Inacio Malmonge Martin ${ }^{1}$, Marcelo Pego Gomes ${ }^{1}$, Marco Antonio Ferro ${ }^{2}$ and Franklin Andrade Silva ${ }^{1}$ \\ 1. Physics Department, Technological Institute of Aeronautics-ITA, São José dos Campos, SP 12228-900, Brazil \\ 2. Aeronautical and Space Institute-IAE, São José dos Campos, SP 12228-900, Brazil
}

\begin{abstract}
Gamma radiation measurements integrated between $200 \mathrm{keV}$ and $10.0 \mathrm{MeV}$ were performed between 03/07/2017 and 05/24/2017 from a tower of 25 meters of altitude in the region of São José dos Campos, SP, Brazil. Throughout this period, there were 9 intense and moderate rains with 11 arrivals of cold fronts coming from southern Brazil. Through measurements of gamma radiation integrated in the energy range mentioned above, the presence of these meteorological parameters and their variations in the region can clearly be observed. Through a potential calibration between the measured gamma radiation intensity and the observed rainfall intensity, it is possible to monitor rains by time interval using this gamma ray detector. Another very important parameter for the region consists of monitoring the number of passages of cold fronts that interfere in the local climatology. This low-cost, easy-to-operate technique can be applied and used in any tropical and equatorial region of the earth's surface.
\end{abstract}

Key words: Ionizing radiation, rain, meteorological parameters.

\section{Introduction}

The ionizing radiation, present on the earth's surface, as well as, the ground/air interface, depends on the presence of cosmic radiation and telluric radiation [1]. Ionizing radiation can also be produced by man, called artificial radiation, for application in industry, medicine and dentistry, as well as, employed in military devices. This artificial radiation and other applications are usually controlled in specific regions or locations [2]. Cosmic radiation is produced on earth as well as its gaseous atmosphere through interactions (particles/photons) of very high energy of extraterrestrial origin with the earth's environment [3, 4]. This radiation produced near the earth's surface is termed secondary cosmic radiation $[5,6]$. It varies depending on the vertical height and latitude of earth. The intensity of this secondary cosmic radiation is

Corresponding author: Inacio Malmonge Martin, Ph.D., main research fields: ionization radiation, solar physics and earth atmosphere. maximum at the poles and minimal at the earth's equator [7]. In relation to the vertical height, it is maximum at $16 \mathrm{~km}$ which is called the Pfotzer maximum and minimum near the surface and at altitudes superior to $40 \mathrm{~km}$. With regard to telluric ionizing radiation, its intensity depends on the regions of the earth's surface. It was formed during the creation of the earth and is present to this day. Its radiation comes from the three series of decay, that is, ${ }^{238} \mathrm{U},{ }^{232} \mathrm{Th}$ and ${ }^{40} \mathrm{~K}[8,9]$. Radon gas (Rn-222) from the set of ${ }^{238} \mathrm{U}$ through its daughters ${ }^{218} \mathrm{Po},{ }^{214} \mathrm{~Pb}$ and ${ }^{214} \mathrm{Bi}$ emit alpha particles and gamma rays at the site. These are the main known sources of the presence of ionizing radiation on the earth's surface $[10,11]$.

\section{Material and Methods}

The gamma ray detector for the energy range of 200 $\mathrm{keV}$ to $10.0 \mathrm{MeV}$ consists of a scintillating crystal of Thallium-doped Sodium Iodide. This crystal is directly coupled to a photomultiplier that records the signals coming from the scintillator and with amplification 


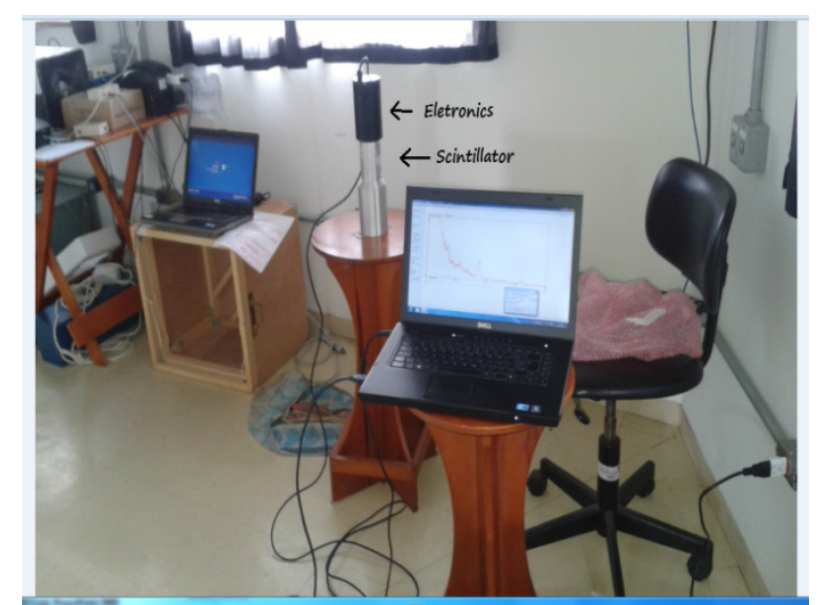

Fig. 1 View of the gamma scintillator with associated electronics and computer.

with a digital analog converter is registered by a computer [12]. This experimental set is seen in Fig. 1 located in the inner room of a tower 25 meters high in relation to the ground.

The scintillator coupled to the photomultiplier is wrapped in a thin layer of aluminum to make it portable. The set (scintillator + associated electronics + data acquisition) only depends on a laptop with a charged battery to measure radiation for up to 5 continuous hours. However, for series of long measurements, electrical grid or photovoltaic energy is used. The scintillator and associated electronics were calibrated in terms of energy and counting intensity per minute at the ITA (Technological Institute of Aeronautics) experimental physics teaching laboratory using radioactive sources.

\section{Results}

Measurements were performed between 07/03/2017 to $24 / 05 / 2017$ at the same location shown in Fig. 1, in the 25-meter high ACA/IAE (Division of Atmospheric Sciences/Aeronautical and Space Institute) tower [13]. The interval between each measurement was set at 1 minute. Therefore, it was possible to verify periods of rain and the dynamic of cold fronts in the region. Fig. 2 shows during this whole time nine rains and eleven intense and moderate cold front passages.

Fig. 3 shows a zoom near the interval of 70,000 minutes after the beginning of the measurements, where a cold front passes in the region culminating with an intense rain caused by that front. During near 5 days, there was a small increase of the local gamma radiation, causing at the end of these five days an intense rain. This established meteorological dynamic is well observed in the region, in this period of time between five and seven days, causing rain and cloud covering less or more intense.

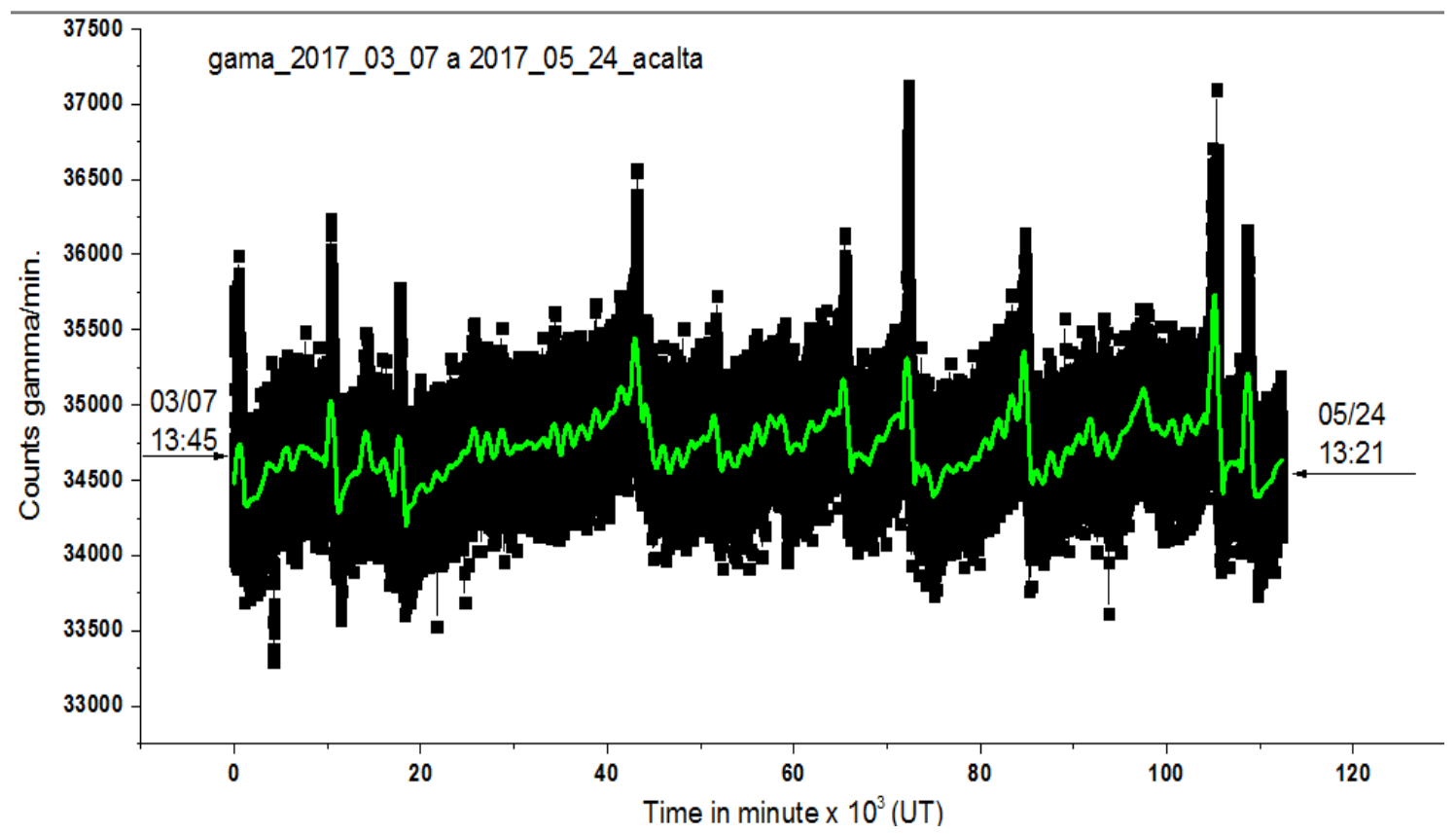

Fig. 2 Measurements of gamma radiation showing rainfall and cold fronts in the period of 03/07/2017 to 05/24/2017. 
Correlation between Rainfall and Cold Fronts with Gamma Ray Measurements near

Ground Level in Southeastern Brazil

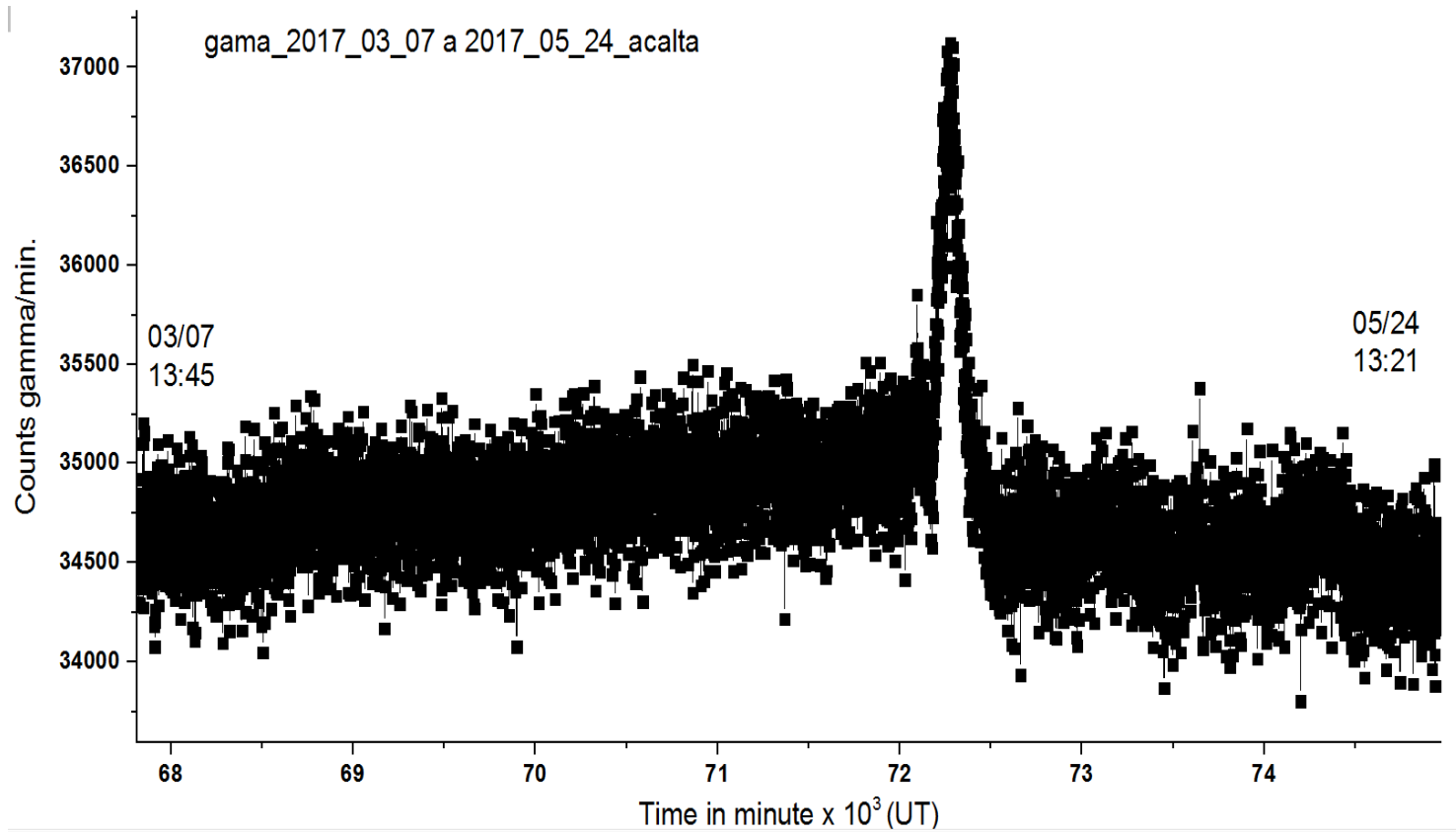

Fig. 3 Radiation dynamic measured during the cold front passage producing an intense rain and drizzle.

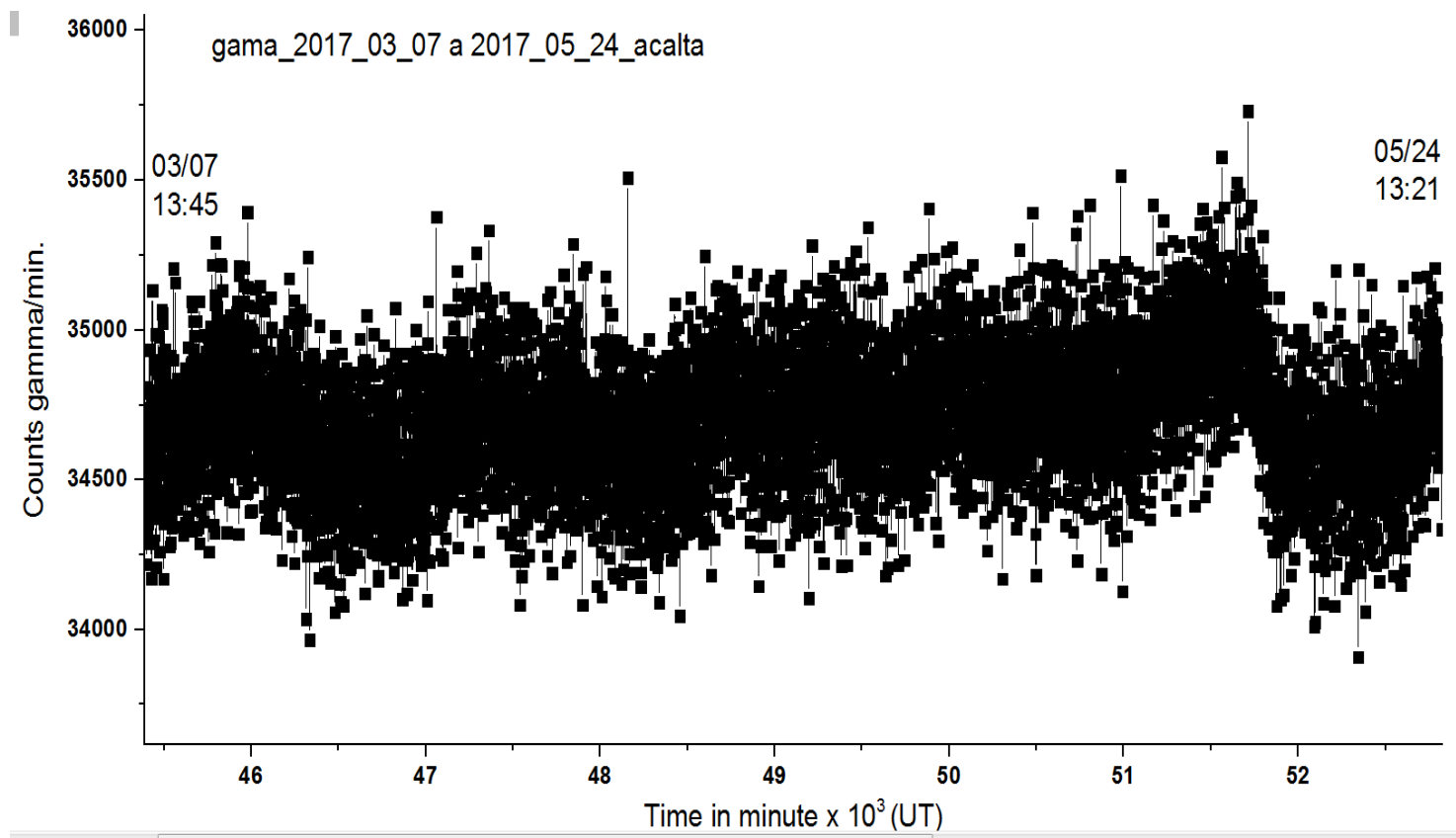

Fig. 4 Cold front passage with change of radiation profile by cloud coverings between 51,500 to 52,000 minutes from the start of measurements.

Fig. 4 shows the dynamic of another cold front that develops and ends only with cloud cover, causing no rain in the region.

A typical case of the formation and passage of the cold front is one that, in the last two days before rain, there is a great increase of local temperature. This meteorological phenomenon causes an increase of gamma radiation during the day and a decrease during the night, as shown in Fig. 5. The day/night cycles are caused by greater or lesser exhalation of the radon gas from the soil, therefore, contributing to a greater or lesser amount of gamma radiation observed at the measurement site.

Between the 84,000 and 86,000 monitoring times, 
seen in Fig. 5, there was cloudy and drizzly weather in the region. This change of state in weather can be seen in the curve of the intensity of gamma radiation measured, as indicated in Fig. 5. On the contrary, when the weather becomes cloudy and it rains every day with varying intensity, the typical curve of radiation measured is shown in Fig. 6. Between the time of measures 104,500 and 105,800 minutes, there are 5 varieties of rains with different intensity.

In the period shown in Fig. 6, the weather pattern was overcast and with drizzles, so the measured radiation curve does not show day/night variation indicating equal exhalation of the radon gas by the terrestrial surface. In Fig. 7, the rainfall spectrum was

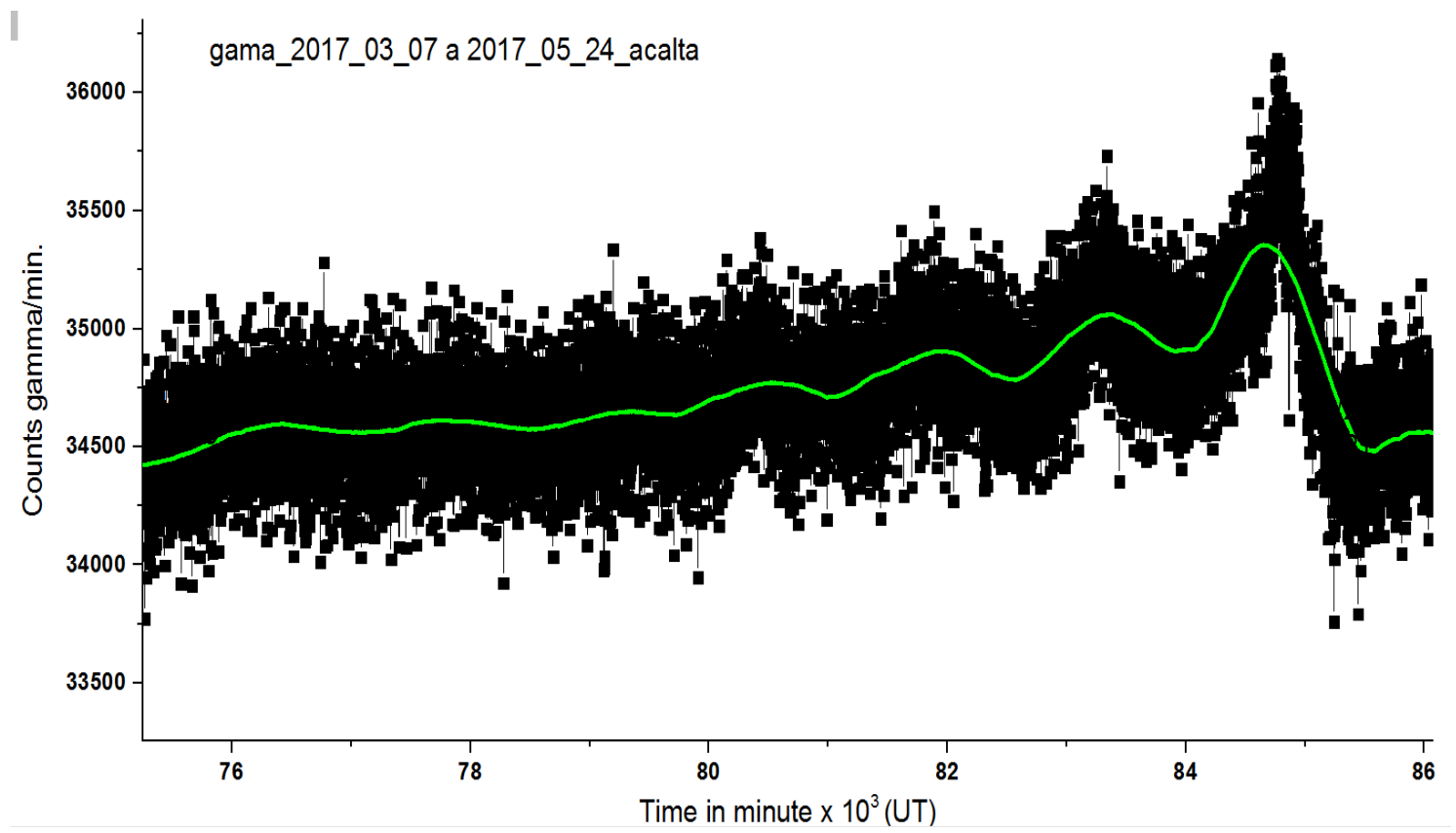

Fig. 5 Day/night variation in the local radiation dynamic due to the increase in temperature before the arrival of the cold front and the rain.

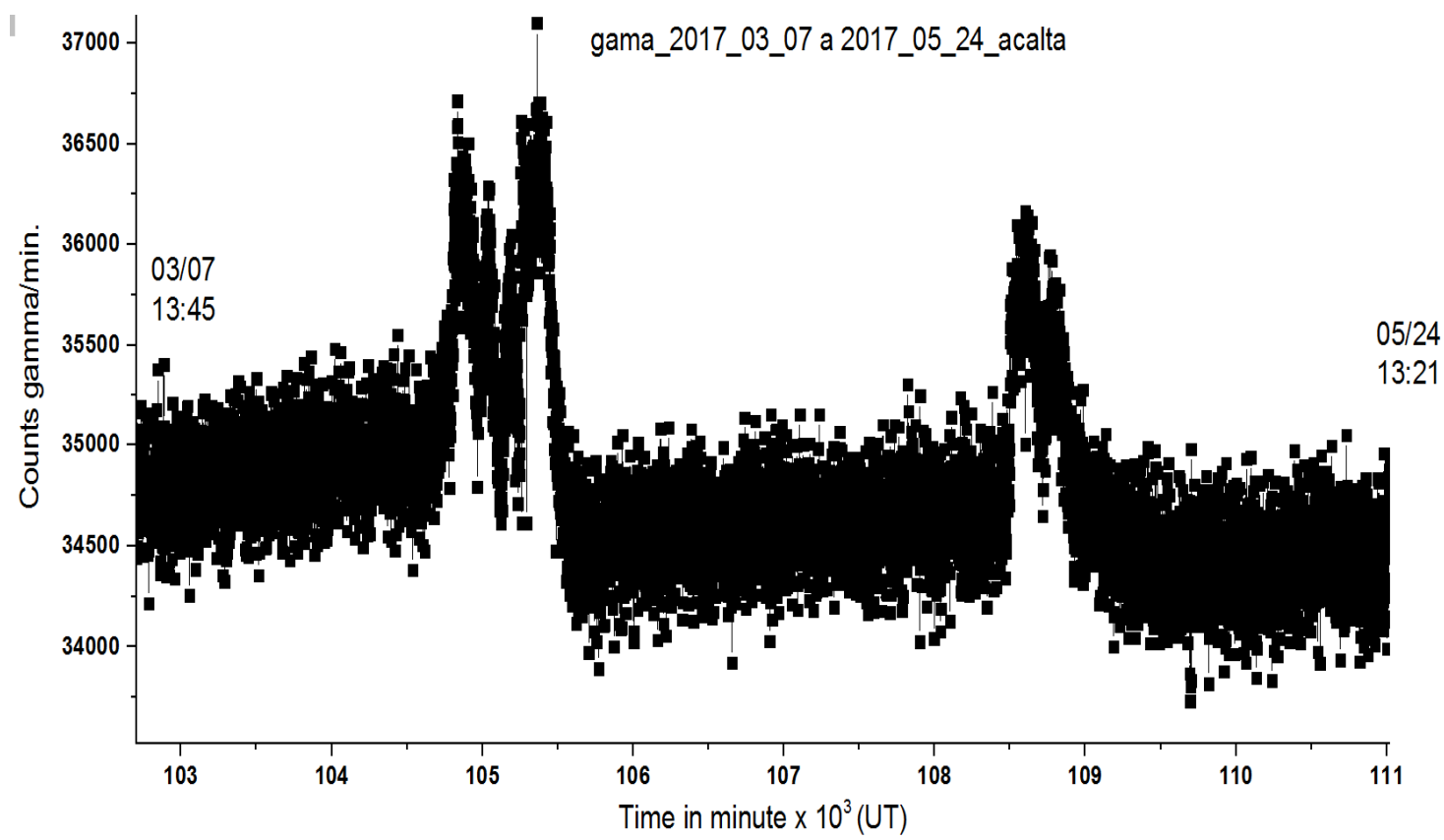

Fig. 6 Rainfall during the whole day and during half a day with different intensities. 


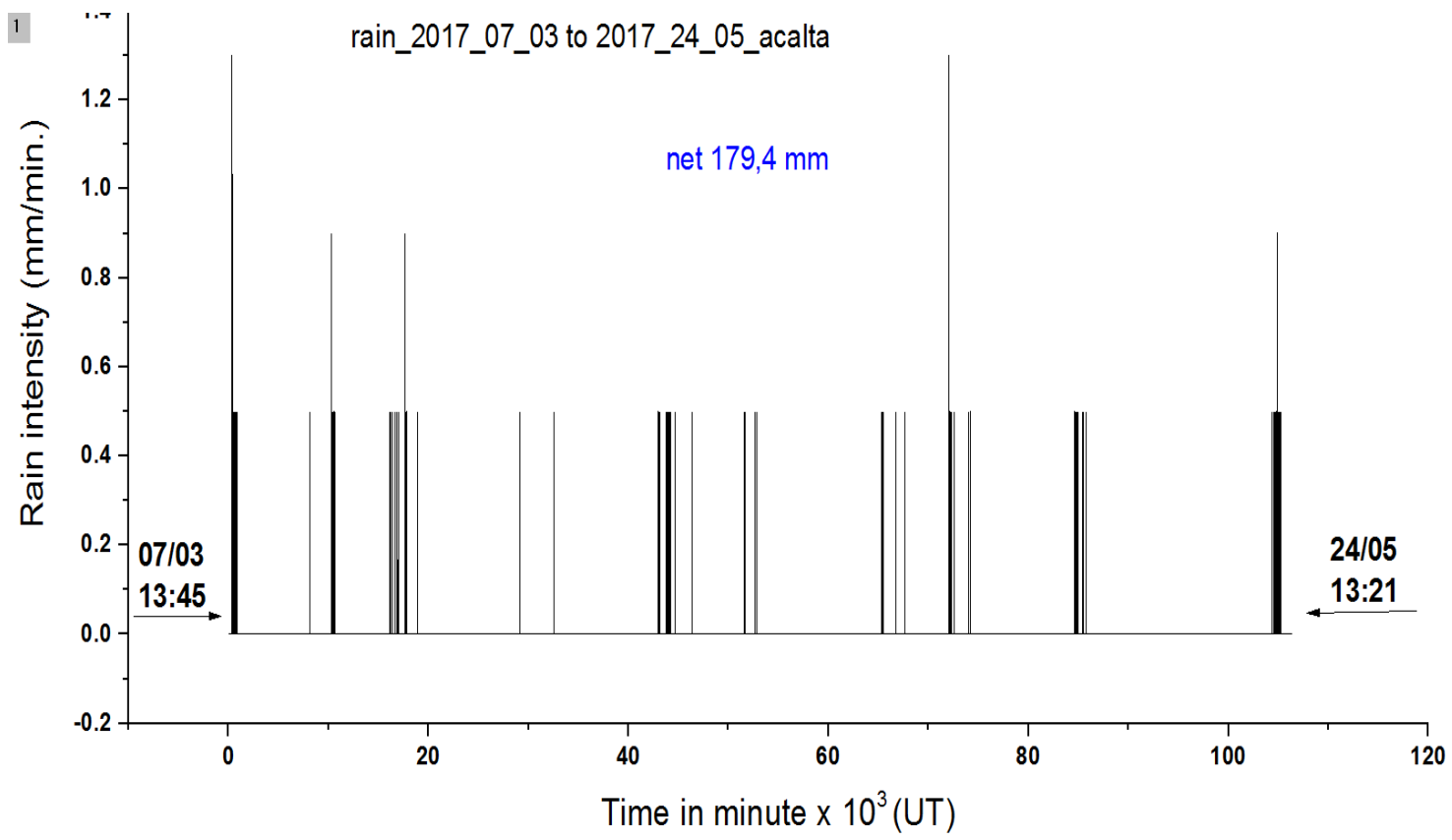

Fig. 7 Rainfall intensity per minute between $03 / 07 / 2017$ and 05/24/2017.

plotted from minute to minute, from 03/07/2017 to $05 / 24 / 2017$, measured at the same location of the gamma radiation detector at the top of the tower. It is thus possible to compare existing rains and variations of gamma radiation measured at the same site.

In Fig. 7, it can be observed that the last rain plotted when expanded, in fact, indicates the presence of two rains of greater intensity than the drizzles, separated by a time of 4 minutes. In terms of measured gamma radiation, this is also evidenced as shown in Fig. 6. Checking Figs. 2 and 6, authors can see a positive correlation between rainfall intensity and gamma-ray intensity variation measured at the same site during 03/07/2017 until 05/24/2017.

\section{Conclusion}

It was determined in this work that the gamma radiation measured between the energy interval of 200 $\mathrm{keV}$ and $10.0 \mathrm{MeV}$ shows the climatic conditions of the region in terms of the passage of cold fronts and rains. This correlation is evidenced by the presence of radon gas (Rn-222), which is in the equatorial and tropical regions exhale from the terrestrial surface because of temperature. On hot and dry days, this gas appears more intense exactly at local noon and less intense at local midnight. During intense rains arriving through cold fronts, the presence of the gas is increased in the lower atmosphere by the washout phenomenon. Continuous rains of low intensity do not allow the exhalation of radon gas from the soil making the radiation constant in that place during that period. In general, the cold fronts, since its formation in the south American continent or southern Brazil until its arrival in the region of São José dos Campos, increase the concentration of local radon gas. The reason for this dynamic of radon gas increase is not yet well explained by researchers in the field. By means of precise measurements using intervals of minutes measuring the intensity of rains and the gamma radiation in the energy range described above, it was possible to experimentally observe the correlation of gamma radiation versus the arrival of cold fronts and rains in the region.

\section{Acknowledgment}

Thanks to CNPq (National Counsel of Technological and Scientific Development) and CAPES (Coordination for the Improvement of Higher Education Personnel) by the fellowships grants support to the group's researchers and the ITA 
Ground Level in Southeastern Brazil

Division of Fundamental Sciences for supporting this research.

\section{References}

[1] Martin, I. M., Gomes, M. P., de Carvalho, R. R. F., and Gomes, R. 2017. "Study of a Portable Experimental Set for the Monitoring of Ionizing Radiation in the Tropical Region of Brazil." Journal of Environmental Science and Engineering A 6 (3): 144-8.

[2] Webster, H. C. 1932. "The Artificial Production of Nuclear y-Radiation." Proceedings of the Royal Society of London. Series A, Containing Papers of a Mathematical and Physical Character 136 (829): 428-53.

[3] Bacioiu, I. 2016. "Study of Extensive Air Showers in the Earth's Atmosphere." Romanian Reports in Physics 68 (4): 1488-94.

[4] Pacini, A. A. 2017. "Cosmic Rays: Bringing Messages from the Sky to the Earth's Surface.” Brazilian Journal of Physics Teaching 39 (1): e1306-1-10.

[5] Grieder, P. K. 2010. Extensive Air Showers (Vol. 1). Berlin: Springer.

[6] Planel, H. 2004. Space and Life: An Introduction to Space Biology and Medicine (Vol. 1). CRC Press.

[7] Bacioiu, I. 2011. "The Theoretical Interpretation of Some Cosmic Rays Reaching the Sea-Level and the Meson Theory." Romanian Reports in Physics 63 (1): 161-71.
[8] Fujinami, N. 2009. "Study of Radon Progeny Distribution and Radiation Dose Rate in the Atmosphere." Japanese Journal of Health Physics 44 (1): 89-94.

[9] Bui-Van, N. A., Martin, I. M., and Turtelli Junior, A. 1988. "Measurements of Natural Radioactivity at Different Atmospheric Depths." Science and Culture Supplement 40 (7): 407.

[10] Martin, I. M., Gusev, A. A., Santos, T. A., and Sismanoglu, B. N. 2015. "Radon Gas and Increasing Intensity of Gamma Radiation near Ground Level Interface on 2011 in Sao Jose dos Campos, SP, Brazil.” International Journal of Research in Engineering \& Technology 3: 31-8.

[11] Babich, L. P., Bochkov, E. I., Kutsyk, I. M., and Rassoul, H. K. 2014. "Analysis of Fundamental Interactions Capable of Producing Neutrons in Thunderstorms." Physical Review D 89 (9): 093010.

[12] Boardman, B. J. 2015. "Aware Electronic Corp.” Accessd May 1, 2017. http://www.aw-el.com/users.html.

[13] Martin, I. M., Alves, M. A., Gomes, M. P., Ferro, M. A., Pinto, M. L., and Antonio, F. C. 2013. "Measurements of $\mathrm{X}$ and Gamma Radiation at Ground Level and Their Correlation with Atmospheric Electric Discharges and Rainfall in São José dos Campos, SP, Brazil.” In 13th International Congress of the Brazilian Geophysical Society \& EXPOGEF, Rio de Janeiro, Brazil, 26-29 August 2013: 272-4. Society of Exploration Geophysicists and Brazilian Geophysical Society. 